\title{
Human-Centric Functional Modeling and Metatheories of Psychology
}

\author{
Andy E. Williams ${ }^{1 *}$ \\ ${ }^{1}$ Nobeah Foundation, Nairobi, Kenya \\ *awilliams@nobeahfoundation.org
}

\begin{abstract}
The newly emerging science of Human-Centric Functional Modeling provides an approach towards modeling systems that is hypothesized to maximize human capacity to understand and navigate complexity in those systems. This paper provides an overview exploring how Human-Centric Functional Modeling might be applied in psychology, and how this increase in capacity to understand complexity might be achieved within the discipline. One potential application is increasing our capacity to compare metatheories of psychology. Given that developing and/or understanding any one particular metatheory of psychology can be a life-long achievement, and assuming that understanding and comparing more than one or two such metatheories might be outside the cognitive capacity of most individuals, one potential key area of usefulness for Human-Centric Functional Modeling is that it provides a single universal way of modeling the human organism and its psychology so that all such metatheories might be compared in each context of their use in order to collectively converge on a single meta-understanding of psychology and of clinical interventions in psychology that best reflect observations.
\end{abstract}

\section{Background}

This paper explores how "state spaces" can potentially be used to model cognition as well as to semantically model theories or metatheories in psychology. There is an established tradition in philosophy of using semantic models for representing scientific theories [1]. While the philosopher Bas C. van Fraassen has been attributed credit [2] for the concept of the "state space" as a semantic modeling approach, a number of researchers citing his work have taken the concept of "state spaces" similar to those described in this paper and applied them to all of science in general, including to psychology as this paper has attempted to do [3]. The difference appears to be that this paper has made the additional refinement of using the human system, particularly the cognitive system, as a basis for this state space modeling approach, so that these models could be understood intuitively by first person introspective observation (by looking "inwards") without the need to understand any logical frameworks at all. Hence the name "Human-Centric Functional Modeling" or HCFM. Since use of this functional state space is predicted to enable us to exponentially increase our ability to understand and navigate complexity in any system it is used to represent and to exponentially increase our ability to solve problems in these systems where not otherwise reliably achievable [28], it follows that using functional state space to model cognition is predicted to significantly increase our capacity to understand and validate theories and metatheories in psychology.

In short, the functional modeling approach proposed here defines a semantic model for information about any and every thing, and therefore defines a model for our thoughts. It also describes a model for the real-world systems (such as cognition) that we can think about. Either of these models might correct or incorrect, and validating each of them is a separate effort. But distinguishing the difference between them likely resolves a number of concerns that this article might give rise to.

Firstly, the conceptual space or "space of concepts" described in this article is not only hypothesized to be a model for thought (concepts and reasoning), but its also hypothesized to be the first complete semantic representation of the meaning of information. Hence it's potentially a model for the way we 
think about "theories," "metatheories," and "models" in psychology or any other science. Whatever ideas one might have about what each of these entities are, such semantic model just provides away of representing them. In this case the semantic model makes no claims about what a theory, metatheory, or model might be in any absolute sense. It just models the relationships with other concepts and lines of reasoning that give those terms meaning to each individual.

Secondly, the functional modeling approach described here was developed to define a model for our cognition, and a model for how our cognition thinks about "theories," "metatheories," "models" and any other concepts or reasoning. The semantic model used by this model of cognition does not interpret any theory at all, it just models the meaning of that theory. It is this model of cognition itself that interprets theories in terms of their impact on properties of cognition relevant to psychology such as cognitive well-being. Once this semantic model is fully developed it is intended to model all theories and metatheories in psychology without assuming any model of cognition at all. And once this model of cognition is fully developed it is intended to be used to test each theory or metatheory of psychology in terms of its fitness in achieving cognitive well-being according to this model of cognition. Where results are inconsistent with observation this permits the model of cognition to be changed more intelligently, perhaps to better match any given theory or metatheory. A model of a system is a kind of "calculator" that can be used to determine the value of properties of that system under certain conditions. Rather than being designed to force all meta theories in psychology to use the conceptual architecture integral to the sort of modeling described here, the goal is simply to create a "calculator" that calculates what understanding is most consistent with the input the calculator has been given (namely the input about all theories or metatheories and data about their effectiveness in achieving each outcome). This approach is very common in systems dynamics. When the number of theories, the amount of data, the precision with which one must consider the theories and data, and the interactions between all of these together become too great, then problem of understanding the truth becomes too complex to be reliably solvable. Dynamical models record all of these constraints so its possible to solve this problem.

One might question whether cognition (thinking) can be readily understood as a kind of movement from one functional state (one concept) to another functional state (another concept). This is a question about whether this functional state space defined in this paper to represent the behavior of the cognitive system (this "conceptual space") is really a complete semantic representation of information. One might also question whether cognition (thinking) can be readily understood as moving through this conceptual space according to some sort of algorithm, perhaps one that selects the path with the least complexity and that reasons with optimal functional efficiency. This is a question about whether the model of cognition defined in this paper is a good model for cognition, or in other words it is a question regarding whether this model of the way the cognitive system moves through that conceptual space is capable of providing a complete representation of the behavior ofcognition. The theories presented in this paper about how a semantic model for information must be constructed in order to be complete might be wrong. Perhaps a semantic model should not represent concepts as transitioning to other concepts through reasoning orunderstanding processes. Perhaps some entities other that concepts and reasoning should be involved. Whether this particular model is wrong or right does not negate the possibility that SOME semantic model might be valid. And if ANY semantic model is valid, all representations oftheories and meta theories defined with that semantic model are valid representations even though they might not be valid theories. The challenge in all cases then reduces to steadily improving the semantic model so that it is valid.

Similarly, the model presented in this paper for how cognition works might be right or wrong. Perhaps the principles driving its operation are very different than the algorithm described here. But whether 
this paper and this particular model is wrong or right does not negate the possibility that SOME model for cognition might be valid. And if ANY model of cognition is valid, then all representations of the fitness of theories and meta theories of psychologies are valid representations according to that model of cognition even though they might not be valid theories. The challenge is again steadily improving the model, in this case the model of cognition, so that it is valid.

In summary, areas in which theories or meta theories are not commensurate might derail many efforts to define such universal models for theories or meta theories. However, because a functional approach to semantic modeling only models the function of that model, and because a functional approach to modeling cognitive behavior only models the function (behavior) of that cognitive model and the fitness of that behavior at achieving a given outcome, a functional approach does not assume any mechanism of causality. Therefore any assumed mechanisms of causality differentiating one meta theory from the next cannot be a source of incomensurability at the level of theory that might derail such an effort.

\section{Introduction}

Human-Centric Functional Modeling is an approach towards modelling living systems [4] such as the human organism, as well as towards modelling inanimate systems. In this approach systems are modelled as having a set of observable behaviours (functions) through which they transition from one functional state to another. These states form a "functional state space" which the system acting in that domain moves through. All the states accessible through those functions (i.e. all the states in the "functional state space") then reflect all possible behaviors of the system, with each system being confined to the states accessible within a given domain of behaviour (i.e. all states of the system belong to some common category). When applied to the cognitive system, this functional state space is a mathematical space that enables all properties of cognition like "intelligence" or "complexity" to potentially be understood in terms of simple mathematical expressions.

Each functional state space used to represent a system in Human-Centric Functional Modelling is defined in terms of a graph containing a network of nodes representing the functional states, connected by edges representing the interaction processes between those states. It has been claimed that "all Complex Systems can be represented and described as networks" [5], that is, as a graph of nodes representing functional states that interact through edges representing transition processes between those states. In the space of concepts or conceptual space that acts as the functional state space of the cognitive system, these nodes are concepts and these processes are reasoning, both of which can by definition be conceptualized. Because the mathematical expressions defining properties of the cognitive system are represented solely in terms of such graphs, these expressions can in many cases be generalized to apply to non-human systems, so that looking inward to gain an understanding of the cognitive system facilitates a deeper understanding of all systems through this human-centric approach.

Through representing all systems as moving through the same abstract mathematical space that the cognitive system is represented as moving through, it is then potentially possible to draw analogies through first person observation of one's own cognitive system that help in understanding other vastly different systems that must be observed through external observation. Applying HCFM potentially involves a massive effort in mapping out this mathematical space representing all possible behaviors of the cognitive system, as well as involving massive efforts in mapping out the mathematical spaces representing other systems the cognition interacts within this human organism, such as the sensorymotor system, the emotional system, and the consciousness system. Using HCFM this effort might be approached methodically, dividing up the conceptual space so that tens of thousands of scientists across many disciplines might be organized to map it. Once such a model of cognition has been mapped out, 
the impacts on a wide variety of disciplines are potentially transformative. For one, representing biological systems in terms of functional state spaces enables it to be seen that nature has already solved problems that can be represented in these abstract spaces as being relevant to a wide range of science and engineering disciplines, as well as being relevant to a great many social and organizational outcomes. Human-Centric Functional Modeling is in essence a method of biomimicry. Namely, achieving general problem-solving ability in any of these abstract mathematical spaces, and hence in the given corresponding real world domain, is believed to be the same general problem that must be solved to address existential challenges from poverty to climate change, where nature has demonstrated those solutions to work for hundreds of millions of years.

Having psychologists validate the potential use of HCFM in representing all metatheories in psychology, as well as in representing all possible theories of change or clinical interventions in each branch of psychology, is one approach towards accomplishing the important step of validating the use of HCFM to model cognition, emotions, consciousness, the body, and all their interactions in different environments, as defined by different models in the various branches of psychology. This step in turn might help validate the use of an HCFM based model of cognition to help understand all other systems from a human-centric perspective.

\section{The Challenges Related to Metatheories in Psychology}

For most scientists and philosophers the word science means first and foremost empirical research, the results of which are descriptions of observations. Others add theoretical thinking as one of the components of science, the role of which should be to produce theories as sets of testable hypotheses along with explanatory models. Others might add philosophical thinking to produce sets of metatheses [6]. In psychology, theoretical psychology can be defined as the "metascientific study of psychological theories and theory-problems" [17].

It has been suggested that "as a discipline, psychology is a giant with feet of clay" [7] because there is little coherence and consensus about the meaning of key psychological terms - including such terms as psychology, mind, or behavior [8]. Though much consideration has been given to the question of "why psychologists hold such contrasting metatheoretical positions" [19], the fact remains that it has also been said that "from its inception, psychology has been characterized by conceptual fragmentation and slow scientific progress" [11], and that in contrast, the natural sciences have achieved remarkable advances and a deepening level of self-consistency in recent decades [12]. However, the quest for unity must be reconciled with the fact that the enormous plurality of knowledge deriving from the multiple paradigms guiding psychotherapy theory, research, and practice might also be deeply valuable [20]. It has been further suggested that several barriers exist against the emergence of a more self-consistent science of psychology and that some metatheories may play an important role in addressing such barriers [13], such as cognitive neuroscience, which is said to provides an empirically testable metatheory and a promising bridge between psychology and the natural sciences [11], [14].

Drawing on evidence marking psychology as a "soft" science, some authors [7] suggest that psychology can profit by adopting as its metatheory the foundation provided by evolutionary psychology. Others argue that basing psychological metatheory on evolutionary psychology is unlikely to solve the problem of incoherence, suggesting instead a unified metatheory must: (a) establish a shared psychological lexicon; (b) elaborate a methodology that coordinates first-, second- and thirdperson modes of inquiry, and (c) develop a process model that describes psychological functioning at the biological, psychological and socio-cultural levels of analysis [8]. 
Evolutionary theory might have the potential to serve as a metatheory for developmental psychology but as currently used in psychology, evolutionary theory is said to be lacking the formal mathematical models that are the norm in evolutionary biology. Formal modeling is believed to be required to tackle the complexity of resolving differences between metatheories through developing families of models and exploring their consequences. By approaching the same question from different angles through doing so, families of models reveal useful generalizations, alongside specific predictions in particular conditions. Or in other words "evolutionary developmental psychology needs to embrace this complexity by representing the full richness of models from biology and developing its own models of human development" [16].

In the future, one might envision a developmental psychology in which evolutionary ideas are formalized and held to the same standards or better as those in biology. Achieving this goal will require evolutionary developmental psychologists to embrace formal theory, as well as invest in training the next generation of scholars to use and understand formal modeling tools. Some argue that evolutionary psychology should be expanded to consider areas such as evolutionary developmental biology if it is to offer new insights regarding the major issues in psychology such as "the domain-specificity of the human mind, the nature-nurture debate, stages in development, and the origin of individual differences" [9], since evolutionary developmental biology provides "evidence for the hypotheses that domain-general and domain-specific abilities co-occur, that nature and nurture interact in a dynamic and nonadditive way, that stages occur in development, and that individual differences are the result of pleiotropic effects during development" [9].

Where Human-Centric Functional Modeling attempts to define a representation of all functions of the human organism that are relevant to any metatheory of psychology, other attempts have also been made to define a single, cohesive framework of functionality to unite the subdisciplines of psychology. The CAPs model for one attempts to characterize individuals by their distinctive pattern and strength of associations among their mental representations, so that one might understand outcomes achieved by that individual in terms of the strength of those associations in different contexts. Such considerations might address, for example, why "seemingly the same social contexts lead one person to smoke, while another person successfully resists the urge to smoke" [18]. Another proposed hierarchical metatheory of psychological science aims to organize evolutionary psychology, evolutionary developmental biology, developmental psychobiology [15], and various subdisciplines of psychology related to various functions in the hierarchy of functionality within the human organism [10]. The difference is that where such approaches pick and choose which functionality to represent according to assumptions of which are relevant to psychology, Human-Centric Functional Modeling is intended to create the capacity to represent all functions of the human organism so that any unforeseen relationships might be discovered as well.

\section{Representing Psychology in Human State Space}

According to Human-Centric Functional Modeling, human beings can potentially be represented as moving through a composite "human state space" consisting of a sensory-motor space navigated by the body, an emotional space navigated by the emotions, a space of concepts or conceptual space navigated by the mind, and a space of awareness's navigated by the consciousness. In any functional state space a problem is defined as the lack of a path from an initial point representing one functional state, to a final point representing another functional state. A solution is a process which defines that path through this functional state space. Therefore any problem in psychology is potentially defined as a gap between two points (two functional states) in that space, and any theory or intervention that solves that problem might be represented as causing the subject to navigate the path between those two points. 
As a concrete example, the conceptual space defined using Human-Centric Functional Modeling provides a representation for all concepts as well as for all reasoning or understanding processes required to navigate between those concepts. These represent all the consciously observable (external) functionality of cognition. In addition to this observed functionality, in Human-Centric Functional Modeling cognition is assumed to require some internal (unconscious) functionality in order to achieve this navigation. This internal functionality is hypothesized to be represented by six functions from the functional perspective [21], but neither these internal functions nor their implementation in terms of biomolecular inputs and output or in terms of structures within the brain or other human organs that might process them, are considered here. The importance of Human-Centric Functional Modeling in psychology is that it potentially allows properties of cognition to be defined mathematically so that ideas in psychology can be explored more objectively and conclusions reached more reliably.

As an example, in this conceptual space a group of concepts is still a concept, though a larger one. A problem, as mentioned, is a gap between two concepts. A solution is reasoning that provides a path from one concept to the other. General problem-solving ability is then hypothesized to be the potential ability to reason from any given problem in conceptual space to any given solution. The magnitude of general problem-solving ability, or intelligence, is hypothesized to be the volume of conceptual space that can be navigated per unit time, multiplied by the density of that conceptual space. A General Collective Intelligence is a hypothetical software platform [22] that organizes individuals into a collective cognition that might have exponentially greater intelligence, and therefore exponentially greater ability to solve any given problem [23] such as understanding which metatheory of psychology is best overall. This definition of intelligence remains to be validated and this capacity to exponentially increase intelligence remains to be confirmed.

As another example of how a functional state space permits the mathematical definition of observable properties of cognition, in this conceptual space the complexity of reasoning is hypothesized to be its length in conceptual space multiplied by the linear density of concepts along that reasoning solution. The complexity of a problem is the complexity of the least complex solution (reasoning) that solves it. In functional state space properties that might be assigned to processes, like a set of physical, emotional, cognitive, or conscious self-awareness processes being assigned by a psychologist to be "maladaptive", potentially all have objective definitions, though these definitions remain to be validated. By making a comprehensive list of all possible cognitive attributes, and by simulating the navigation of the cognitive system through that conceptual space, a representation of conceptual space which possesses those attributes might be verified as behaving in a manner consistent with those definitions. By contrast, the Diagnostic and Statistical Manual of Mental Disorders (DSM-5) [24] has been criticized as being more a political document than a scientific one.

Attribute of Cognition Interesting/Uninteresting

Attentive/Inattentive

Perspective

\section{Potential Representation in Conceptual Space}

Level of interest is proposed to be an attractive force that causes concepts to move in conceptual space towards a concept of interest. If so then level of interest can be represented in conceptual space by differences in density.

Attention is proposed to be a property of the consciousness. Focus of conscious awareness on concept A causes concept A to move in conceptual space in the direction of the problem the cognitive system has placed on being solved (the problem of finding a reasoning path to navigate to concept B). If so then attention can be represented in conceptual space by the resulting motion of concepts over time. Perspective is proposed to be a point in conceptual space from which 
Abstract/Specific

Reasonable/Unreasonable

True/False

Complex/Simple

Resolvable/Unresolvable

Intelligent/Stupid

Intuitive/Methodical

Biased/Unbiased

Definable/Undefinable

Solvable/Unsolvable

Critical/Supportive

Discriminating/

Indiscriminate

Semantic

Reasoning/Understanding reasoning relationships can be navigated.

Level of abstraction is proposed to be the size of the region in conceptual space that encloses concepts with a smaller extent in that space (more specific concepts).

Unreasonable reasoning follows a reasoning path that is inconsistent with multiple other reasoning paths that exist in the conceptual space.

Truth (or systemic error [26]) is proposed to be determined by recurring patterns of reasoning paths in conceptual space.

Reasoning that is complex contains many steps at potentially a high resolution. Simple reasoning contains few steps at potentially a low resolution.

Resolvable problems or solutions contain concepts that can be located within the conceptual space. Unresolvable problems or solutions contain concepts that require greater resolution than the conceptual space has . Intelligent reasoning has the capacity to navigate complex paths at high resolution. Stupid reasoning lacks the capacity to navigate anything but simple paths at low resolution.

Intuitive (type 1) reasoning is a direct path formed between one concept to another that is based on patterns of past reasoning. Rational methodical (type 2) reasoning is a path between one concept and another that is based on some process of computation.

Biased reasoning is strongly predisposed to navigate certain paths in conceptual space when located at a given concept in conceptual space. Undefinable problems contain concepts outside the accessible conceptual space and/or concepts that are unresolvable.

Unsolvable problems contain concepts outside the accessible conceptual space and/or concepts that are unresolvable.

Critical reasoning seeks to find reasoning that proves that the reasoning currently being considered is false by finding reasoning paths consistent with that reasoning that are untrue.

Discriminating reasoning accepts reasoning without evaluating whether it is true.

Conceptual space must provide a description of every region or subregion representing a concept that is fully self-contained within that region. That is, descriptions must be semantic.

Reasoning is the process of navigating the conceptual space.

Understanding is the process of being navigated through the conceptual space.

Table 1: Attributes with which cognitive processes (reasoning or understanding) are executed.

If and when all psychological processes and interventions can be represented as paths through the functional state space of the human system (the "human state space"), then both psychological ailments and psycho-therapeutical interventions might be represented as perturbations of more optimal paths, allowing all such ailments to be semantically modeled, as well as measured, and allowing all such treatments to be applied in an unambiguous way. Once diagnostic, intervention, and other processes can more unambiguously be represented in terms of such paths through a human state space that specifies the state of the entire human system (i.e. that includes the sensory-motor system or 'the body', the emotional system, the cognitive system, and the conscious self-awareness), this might enable the collective understanding of psychological health or wellness to surpass limitations set by the inability 
of groups to reliably exchange concepts as the complexity of those concepts increases. However, case studies must also be performed to validate that this conceptual model of a "human state space" is complete and consistent with current knowledge. Furthermore, software tools must be created to translate natural human language descriptions of psychological health and wellness interventions (i.e. to translate clinical notes) to and from this semantic model. This is not a short-term objective. However, the long-term benefit of using Human-Centric Functional Modeling to define objective functional models for properties such as intelligence is that doing so might allow sharing and convergence of ideas across a far wider range of disciplines.

Human-Centric Functional Modeling might also be useful to psychology in maximizing our collective capacity to make use of every theory or metatheory in psychology if it can be assumed that every intervention that might be achieved through any theory or any clinical intervention in any branch of psychology is both represented in terms of its impact as a perturbation on paths through conceptual space, and is represented semantically in terms of its meaning as a path through conceptual space. Through modeling all such interventions and by measuring their fitness to achieve outcomes in the context of each situation, these interventions might be added to a library that an intelligent agent might use to significantly increase the capacity of a clinical psychologist to achieve positive psychological impact on a given individual, or that a General Collective Intelligence might use to significantly increase the profession's collective capacity to achieve psychological impact across all individuals. In other words, rather than clinical psychologists relying on their education about specific methodologies, where that education must be constantly updated, or rather than clinical psychologists having to search the literature for new developments as well as for guidance regarding whether those developments are widely accepted, instead clinical psychologists might rely upon an instantaneously updating selfsearching, and self-validating database that is vastly larger and more powerful.

Since a representation for functional state spaces as graphs has not yet been fully elaborated, properties like the exact distances between functional states have not yet been computed. In the case of functional state spaces like the conceptual space navigated by the cognitive system, this means that properties such as general problem-solving ability (intelligence) that require the definition of distance cannot be precisely calculated as of yet. However, because distances in functional state space potentially impact our collective problem-solving capacity in a very wide range of fields from physics, to mathematics, to computer science and engineering, to biology, to sustainable development, and because doing so might exponentially increase our general problem-solving ability in these fields [23], then a group of scientists much larger than the community of psychologists can potentially be incentivized to participate in solving these common "representation problems".

In the interim while "definition problems" such as defining mathematical expressions for all of the properties of cognition involved in a given metatheory of psychology, and while "representation problems" such as defining the meaning of distances in conceptual space, have not been solved, and in the interim in which a map of human state space doesn't yet exist for any given individual, HCFM still has a number of uses. Similar to the case in which even before the human genome was sequenced, the concept of sequencing the human genome was still useful in discovering how such a catalog might make it much easier to establish connections between particular genes and disease, it is also true that even before such a map of the human mind is constructed, the concept of mapping the human mind is useful in discovering how such a catalog of processes might make it much easier to establish connections between particular cognitive constructs and psychological wellness or disease. In particular, HCFM might find some usefulness in being used to define simple mathematical models of some properties of cognition, as well as to define the meaning of the various branches of psychology in terms of the human state space, so that these definitions might be validated as well as used to provide 
more objective clarity that enables divergent ideas about those concepts to more reliably converge on a single collective understanding, where currently the concepts can't be resolved sufficiently to do so. In addition, in the early stages such a cognitive map might be approximated in order to be employed in differentiating the various metatheories of psychology.

Modeling the Childhood Development and Adult Evolution of an Individual Human's Psychology If every theoretical model of any psychological process is represented by a set of paths through some functional state spaces within the human system, as is every psychological intervention, then the childhood development and change over adulthood of the conceptual space and the other functional state spaces occupied by the human organism would be expected to fall into concrete categories. For example, assuming that cognitive psychology can be restricted to the cognitive system, theories and interventions in cognitive psychology would be expected to be confined to the conceptual space.

Assuming that developmental psychology concerns the development of the cognitive system both on its own as well as in response to physical development, emotional development, and development of conscious self-awareness, then developmental psychology would be expected to involve the development of all these functional state spaces. Whether talking about evolutionary psychology, which would be expected to involve the evolution of these functional state spaces over evolutionary time scales, neuropsychology, which would be expected to have the capacity to incorporate all possible models of neurological processes, or occupational psychology, which would be expected to have the capacity to incorporate all processes of group psychology represented as occupying the collective functional state spaces, all of these branches in psychology would be expected to gain from the ability to represent their data in this single universal way provided by Human-Centric Functional Modeling, which again appears to satisfy the definition of a complete semantic representation that could potentially enable all other branches to use the same data.

\section{Research Limitations}

Interdisciplinary work faces many barriers. It has been said that "the literature is replete with descriptions of the traditional and persistent barriers to interdisciplinary research, including attitudinal resistance, differing research methods and communication barriers among disciplines, the length and depth of training in a single field necessary to develop scientists who will be successful in competing for funds, the difficulty in forging a successful career path outside the single disciplinary structure, impediments to obtaining research funding for interdisciplinary research, the scarcity of interdisciplinary departments in academe, and the perceived lack of outlets for the publication and dissemination of interdisciplinary research results. The heterogeneity of institutions, structures, and value systems at the private, state, and federal levels compounds the complexity of these obstacles" [25]. Since Human-Centric Functional Modeling aims to maximize interdisciplinarity by defining a single human-centric perspective, it's expected that this approach might maximize those barriers. The key limitation of this research then might not be any limitation to the validity of the research itself, but instead the challenge of communicating that validity. Philosophers have long asked whether if a tree falls in a forest and no one is around to hear it, does it make a sound [27]? In the case of sufficiently interdisciplinary research, the question is especially relevant.

\section{Future Directions}

Future work may explore some specific topics such as how to create self-organizing and self-sustaining processes that might exponentially increase the number of psychologists that can be included in a research team, in order to improve the uptake of any evidence-based research conducted through such a General Collective Intelligence based approach. Another might be to explore how to exponentially increase capacity to achieve a given clinical outcome. Yet another might be to exponentially improve 
capacity to understand and assess the truth of metatheories of psychology. Other potential areas of exploration are below:

- Increasing Research and Clinical Outcomes: Human-Centric Functional Modeling suggests that an exponential increase in ability to solve any problem, and therefore an exponential increase in ability to achieve any targeted outcome in psychology is possible through General Collective Intelligence. Whatever the specialization in psychology being considered, one future direction is to explore what an increase in ability to achieve targeted outcomes in that area of psychology means, how can it be quantified, and how General Collective Intelligence might be used to help achieve it. This research might consider questions such as "How does one achieve a much larger impact of psychological interventions on a much larger number of people?"

- Increasing Ability to Differentiate metatheories of Psychology: One metatheory of psychology might increase ability to validate or invalidate theories of psychology, or to achieve other psychology related outcomes more successfully than others. Whatever the metatheory being considered, one future direction is to explore what an increase in ability to achieve these targeted outcomes in psychology means, how can it be quantified, and how General Collective Intelligence might be used to help achieve it. This research might consider questions such as what HCFM and GCI as theories suggest is required to achieve an exponential increase in that ability.

- Increasing Ability to Achieve Clinical Outcomes with metatheories of Psychology: One metatheory of psychology might increase ability to execute clinical psychology interventions more successfully than others. One future direction is to explore how General Collective Intelligence might be used to help better clinical outcomes.

- Increasing Ability to Overcome Barriers to the Dissemination of Evidence Based Research: One future direction is to explore what are the barriers against increasing the number of psychologists that can be included in an effective research team, what an increase in ability to achieve improve the uptake of evidence-based research through increased team size means, how can it be quantified, and how General Collective Intelligence might be used to help achieve it.

\section{Conclusions}

Semantically modeling theories in psychology, mapping them to the human state space, and ranking them according to their impact with respect to the human state space is a massive task. Before this task can be achieved, it's important to first communicate the possibilities that might be presented in using Human-Centric Functional Modeling to do so, to encourage others to validate the feasibility of the approach in this and other disciplines, until the value proposition is accepted in a large enough range of disciplines to reliably motivate that effort.

\section{References}

[1] Suppe, Frederick. The semantic conception of theories and scientific realism. University of Illinois Press, 1989.

[2] Lloyd, Elisabeth A. "A semantic approach to the structure of population genetics." Philosophy of Science 51.2 (1984): 242-264.

[3] Niiniluoto I. (1987) Quantities, State Spaces, and Laws. In: Truthlikeness. Synthese Library (Studies in Epistemology, Logic, Methodology, and Philosophy of Science), vol 185. Springer, Dordrecht. https://doi.org/10.1007/978-94-009-3739-0_3 
[4] Andy E. Williams, A Revolution in Systems Thinking?, Proceedings of the World Organization of Systems and Cybernetics (WOSC) 2021 Congress, Accepted (2021), link

[5] Newman MEJ (2010) Networks: an introduction. Oxford University Press, New York

[6] Madsen K.B. (1985) Psychological Metatheory. In: Madsen K.B., Mos L.P. (eds) Annals of Theoretical Psychology. Springer, Boston, MA. https://doi.org/10.1007/978-1-4613-2487-4_1

[7] Mascolo, M.F. Inching Toward a Unified Metatheory for Psychology. Integr. psych. behav. 55, 198211 (2021). https://doi.org/10.1007/s12124-020-09543-2

[8] Zagaria, A., Ando', A., \& Zennaro, A. (2020). Psychology: A giant with feet of clay. Integrative Psychological \& Behavioral Science.

[9] Annemie Ploeger, Han L. J. van der Maas \& Maartje E. J. Raijmakers (2008) Is Evolutionary Psychology a Metatheory for Psychology? A Discussion of Four Major Issues in Psychology From an Evolutionary Developmental Perspective, Psychological Inquiry, 19:1, 1-18, DOI: $10.1080 / 10478400701774006$

[10] Badcock PB. Evolutionary Systems Theory: A Unifying Meta-Theory of Psychological Science. Review of General Psychology. 2012;16(1):10-23. doi:10.1037/a0026381

[11] Rand, K.L. and Ilardi, S.S. (2005), Toward a consilient science of psychology. J. Clin. Psychol., 61: 7-20. https://doi.org/10.1002/jclp.20088

[12] Wilson, G.T. (1996). Empirically validated treatments: Reality and resistance. Clinical Psychology:Science and Practice, 3, 241-244.

[13] Henriques, G. (2004). Psychology defined. Journal of Clinical Psychology, 60, 1207-1221.

[14] Ilardi, S.S. and Feldman, D. (2001), The cognitive neuroscience paradigm: A unifying metatheoretical framework for the science and practice of clinical psychology. J. Clin. Psychol., 57: 1067-1088. https://doi.org/10.1002/jclp.1124

[15] Gilbert Gottlieb (2001) The Relevance of Developmental-Psychobiological Metatheory to Developmental Neuropsychology, Developmental Neuropsychology, 19:1, 1-9, DOI: 10.1207/S15326942DN1901_1

[16] Frankenhuis, W.E. and Tiokhin, L. (2018), Bridging Evolutionary Biology and Developmental Psychology: Toward An Enduring Theoretical Infrastructure. Child Dev, 89: 2303-2306. https://doi.org/ $\underline{10.1111 / \text { cdev.13021 }}$

[17] K.B. Madsen, Theoretical Psychology: a Definition and Systematic Classification11The main part of this paper is selected from K. B. Madsen, Psychological Metatheory: An Introduction to Volume 3. Annals of Theoretical Psychology (Vol. 3). New York: Plenum Press, 1985. Republished with permission., Editor(s): William J. Baker, Michael E. Hyland, Hans Van Rappard, Arthur W. Staats, Advances in Psychology, North-Holland, Volume 40, 1987, Pages 165-174, ISSN 0166-4115, ISBN 9780444701206, https://doi.org/10.1016/S0166-4115(08)60071-0.9 
[18] Shoda, Y. and Mischel, W. (2006), Applying Meta-theory to Achieve Generalisability and Precision in Personality Science. Applied Psychology, 55: 439-452. https://doi.org/10.1111/j.1464$\underline{0597.2006 .00264 . x}$

[19] Conway, J. B. (1992). A world of differences among psychologists. Canadian Psychology/Psychologie canadienne, 33(1), 1-24. https://doi.org/10.1037/h0078692

[20] Anchin, J. C. (2008). Pursuing a unifying paradigm for psychotherapy: Tasks, dialectical considerations, and biopsychosocial systems metatheory. Journal of Psychotherapy Integration, 18(3), 310-349. https://doi.org/10.1037/a0013557

[21] Williams, A. E. Approximating an Artificial General Intelligence or a General Collective Intelligence, International Journal of Collaborative Intelligence, in press (2021)

[22] Williams, A. E., Defining a Continuum from Individual, to Swarm, to Collective Intelligence, to General Collective Intelligence, International Journal of Collaborative Intelligence, in press (2021)

[23] Williams, A. E. (2021, October 2). Using Systems Thinking to Exponentially Increase Impact. In review (2021), Retrieved from osf.io/preprints/africarxiv/dcjyf

[24] Krueger, Robert F., and Kristian E. Markon. "The role of the DSM-5 personality trait model in moving toward a quantitative and empirically based approach to classifying personality and psychopathology." Annual review of clinical psychology 10 (2014): 477-501.

[25] Institute of Medicine (US) Committee on Building Bridges in the Brain, Behavioral, and Clinical Sciences; Pellmar TC, Eisenberg L, editors. Bridging Disciplines in the Brain, Behavioral, and Clinical Sciences. Washington (DC): National Academies Press (US); 2000. 3, Barriers to Interdisciplinary Research and Training. Available from: https://www.ncbi.nlm.nih.gov/books/NBK44876/

[26] Gregg Henriques, Andy E. Williams, General Collective Intelligence and Systemic Error in Groups, retrieved from https://www.psychologytoday.com/us/blog/theory-knowledge/202106/generalcollective-intelligence-and-systemic-error-in-groups, on July 19, 2021.

[27] Napier, Jemina. "If a tree falls in a forest and no one is there to hear it, does it make a noise." Advances in Interpreting Research: Inquiry in action (2011): 121-152.

[28] Williams, A. E. (2020, July 11). Human Intelligence and General Collective Intelligence as Phase Changes in Animal Intelligence. https://doi.org/10.31234/osf.io/dr8qn 